\title{
The systemic inflammation response index predicts survival and recurrence in patients with resectable pancreatic ductal adenocarcinoma
}

This article was published in the following Dove Press journal: Cancer Management and Research

\author{
Shuo $\mathrm{Li}^{1-4, *}$ \\ Huaxiang $X u^{1-4, *}$ \\ Wenquan Wang ${ }^{1-4}$ \\ Heli Gao ${ }^{1-4}$ \\ $\mathrm{HaO} \mathrm{Li}^{\mathrm{I}-4}$ \\ Shirong Zhang ${ }^{1-4}$ \\ Jinzhi $X u^{1-4}$ \\ Wuhu Zhang ${ }^{1-4}$ \\ Shuaishuai $\mathrm{Xu}^{\mathrm{I}-4}$ \\ Tianjiao $\mathrm{Li}^{\mathrm{I}-4}$ \\ Quanxing $\mathrm{Ni}^{1-4}$ \\ Xianjun $\mathrm{Yu}^{\mathrm{I}-4}$ \\ Chuntao $\mathrm{Wu}^{\mathrm{I}-4}$ \\ Liang Liu ${ }^{1-4}$
}

'Department of Pancreatic Surgery, Fudan University Shanghai Cancer Center, Shanghai 200032, People's Republic of China; ${ }^{2}$ Department of Oncology, Shanghai Medical College, Fudan University, Shanghai 200032, People's Republic of China; ${ }^{3}$ Shanghai Pancreatic Cancer Institute, Shanghai 200032, People's Republic of China; ${ }^{4}$ Pancreatic Cancer Institute, Fudan University, Shanghai 200032, People's Republic of China

*These authors contributed equally to this work

Correspondence: Chuntao Wu; Liang Liu Department of Pancreatic Surgery, Fudan University Shanghai Cancer Center, 270

Dong An Road, Shanghai 200032, People's

Republic of China

Tel +862164175590

Fax +86216403 I446

Email liuliang@fudanpci.org;

wuchuntao@fudanpci.org
Purpose: The systemic inflammation response index (SIRI), based on peripheral neutrophil, monocyte, and lymphocyte counts, was recently emerged and used as a novel tool in predicting prognosis in different types of cancer. Our aim was to investigate the clinical significance of preoperative SIRI in patients with resectable pancreatic ductal adenocarcinoma (PDAC).

Materials and methods: The SIRI was developed in a training cohort of 371 PDAC patients undergoing radical surgery between 2010 and 2013 and validated in a validation cohort of 310 patients from 2014 to 2015. Baseline clinicopathologic characteristics, preoperative laboratory parameters and follow-up information were collected. The optimal cutoff value of SIRI was determined by receiver operating characteristic curve. Univariate and multivariate analysis were performed to analyze the prognostic value of SIRI.

Results: The optimal cutoff value of SIRI stratified patients into low SIRI group $(\leq 0.69)$ and high SIRI group (>0.69). Survival analysis showed that the median overall survival (OS) and recurrence-free survival (RFS) were significantly better in patients with low SIRI. The SIRI was an independent predictor of OS and RFS in multivariate analysis. In addition, SIRI remained its prognostic significance both in patients with early-stage diseases and in patients with normal carbohydrate antigen 19-9 levels. High SIRI indicated poor treatment response for patients who received postoperative adjuvant chemotherapy.

Conclusion: Preoperative SIRI was an independent prognostic indicator of poor outcomes in PDAC patients after radical resection. It might assist clinicians to identify high-risk patients and choose the optimal individualized treatment strategy.

Keywords: pancreatic ductal adenocarcinoma, systemic inflammation response index, overall survival, recurrence-free survival, prognosis

\section{Introduction}

Pancreatic cancer is a common malignant tumor characterized by fast progress and poor prognosis. It is now the fourth leading cause of cancer-related mortalities in the United States, and the overall 5-year survival rate is approximately $8 \%{ }^{1}$ In China, the incidence of pancreatic cancer has increased sharply in the past few years, and it now ranks the ninth among all causes of cancer death. ${ }^{2}$ Surgical resection remains the only potential curative treatment. However, due to the high incidence of postoperative recurrence and metastasis, the 5-year survival rate for patients after radical surgery is only around $25 \% .^{3}$

Currently, the most reliable predictor of survival for patients with pancreatic cancer is the TNM staging system. This prognostic method mainly depends on 
anatomical features of tumor specimen, and thus can only be available for evaluation after surgery. However, a considerable proportion of pancreatic cancer patients are diagnosed at advanced or late inoperable stages, often making radical resection impossible. In addition, patients with the same resection procedure and therapy procedure usually have different outcomes, which brings difficulties to clinicians when making treatment strategies. Therefore, more effective preoperative biomarkers should be identified to provide more reliable prognostic information for pancreatic cancer patients.

It is widely recognized that local immune response and systemic inflammation plays an important role in the initiation, development, and progression of many solid tumors. ${ }^{4}$ The routine immune and inflammatory cells such as neutrophils, lymphocytes, and monocytes can promote the intravasation of tumor cells into the circulation system and the formation of distant metastasis, which contributes to the poor outcomes of patients. ${ }^{5}$ In clinical practice, systemic inflammation is directly indicated by peripheral blood counts of immune and inflammatory cells and acute-phase proteins such as C-reactive protein. New inflammation indexes based on the integration of these conventional inflammatory parameters, such as neutrophil to lymphocyte ratio (NLR), platelet to lymphocyte ratio (PLR), prognostic nutritional index (PNI), and systemic immune-inflammation index (SII), have shown potential prognostic values in several studies. ${ }^{6-9}$

It has recently been reported that systemic inflammation response index (SIRI), based on peripheral neutrophil, lymphocyte and monocyte counts, can be applied to predict the survival in different types of tumor. ${ }^{10-13}$ Neutrophils can aid tumor cells to evade immune surveillance and adhere to metastatic target organs. ${ }^{14}$ They also facilitate tumor angiogenesis by producing proangiogenic factors. ${ }^{15}$ Meanwhile, neutrophils can secrete large amounts of reactive oxygen species and nitric oxide, both of which lead to disorders of T-cell activation. ${ }^{16}$ Tumor-associated macrophages (TAMs) are derived from circulating monocytes, which can be recruited to the tumor tissue and promote the growth and migration of the tumor. ${ }^{17}$ In addition, they also induce the apoptosis of activated $\mathrm{T}$ cells and accelerate the formation of new tumor vessels. ${ }^{18}$ Peripheral monocyte counts may reflect the level of TAMs, and higher monocyte counts are considered to be negative markers for tumors. Lymphocytes are crucial components of the immune system, serving as the main defense against cancer cells. They control tumor progression by releasing cytokines such as interferon- $\gamma$ and tumor necrosis factor- $\alpha .{ }^{19}$ Downregulation of peripheral lymphocytes impairs host's anticancer immunity and increases the incidence of tumor cell dissemination. The index SIRI combines the above cell types and reflects the complex interplay between immune and inflammatory cells in the tumor microenvironment. However, the prognostic significance of SIRI in operable pancreatic cancer is still unclear to date. The purpose of this study was to examine the prognostic ability of SIRI using two independent cohorts of patients with pancreatic ductal adenocarcinoma (PDAC) who underwent radical surgery.

\section{Materials and methods}

\section{Patient selection}

We retrospectively recruited 371 patients who were diagnosed with PDAC and underwent radical surgery from January 2011 to December 2013 at our institution as the training cohort. To verify the prognostic value of SIRI, we recruited another 310 patients treated at our institution from January 2014 to December 2015 as the validation cohort. The inclusion criteria included: 1) primary PDAC and negative resection margin confirmed by histopathological examination; 2) had measurement of routine blood test and liver function test within 7 days before surgery; 3 ) had complete clinicopathologic and postoperative follow-up data. The exclusion criteria included: 1) received preoperative antitumor treatment; 2) had history of other malignant tumors; 3) clinical evidence of infection or the use of anti-inflammatory or immunosuppressive drugs; 4) perioperative death caused by severe surgical complications. This study was approved by the Human Research Ethics Committee of Fudan University Shanghai Cancer Center and was in accordance with the tenets of the World Medical Association Declaration of Helsinki. Written informed consent was obtained from all patients according to the committee's regulations.

\section{Data collection}

The following clinicopathologic variables were collected in this study: age, gender, tumor location, tumor size, lymph node status, TNM stage, tumor differentiation, vascular invasion, perineural invasion, carbohydrate antigen 19-9 (CA199), chemotherapy and five inflammation-based scores, including NLR, PLR, PNI, SII and SIRI. The clinical staging was determined by TNM staging system of the American Joint Commission on Cancer (AJCC) 8th edition via clinical evaluation and postoperative pathological examination. Blood samples for routine blood test and liver function test 
were obtained within 1 week before surgery and detected in laboratory department. Five inflammation-based scores were defined as follows: 1) $\mathrm{NLR}=\mathrm{N} / \mathrm{L}$; 2) $\mathrm{PLR}=\mathrm{P} / \mathrm{L} ; 3$ ) $\left.\left.\mathrm{PNI}=\operatorname{albumin}(\mathrm{g} / \mathrm{L})+5 * \mathrm{~L}\left(10^{9} / \mathrm{L}\right) ; 4\right) \mathrm{SII}=\mathrm{P} * \mathrm{~N} / \mathrm{L} ; 5\right)$ $\mathrm{SIRI}=\mathrm{N}^{*} \mathrm{M} / \mathrm{L}$, where $\mathrm{N}, \mathrm{L}, \mathrm{P}$, and $\mathrm{M}$ refer to peripheral counts of neutrophils, lymphocytes, platelets, and monocytes. The optimal cutoff values of NLR, PLR, PNI, SII, and SIRI for overall survival (OS) were 2.24, 100.25, 35.15, 330.30 , and 0.69 , respectively, calculated by applying receiver operating characteristic (ROC) analysis.

\section{Follow-up}

All patients were regularly followed up after surgery. Physical examinations were performed, and peripheral tumor biomarkers were measured for each patient every 3 months. Enhanced abdominal computed tomography (CT) or magnetic resonance imaging (MRI) were routinely performed every 6 months. If local recurrence or distant metastasis was suspected, image examinations including CT, MRI, bone scans, and positron emission tomography-computed tomography (PET-CT) were selectively conducted immediately. OS was defined as the interval between the date of surgery and death or the last follow-up visit. Recurrence-free survival (RFS) was defined as the interval between the date of surgery and tumor recurrence or the last follow-up visit. The last follow-up time was October 2017.

\section{Statistical analysis}

All statistical analyses were performed using SPSS 21.0 (Chicago, IL, USA). The optimal cutoff values of NLR, PLR, PNI, SII, and SIRI were determined by ROC analysis. The correlations between SIRI and clinicopathologic variables were analyzed by Pearson Chi-squared test. Cox regression methodology was used for univariate analysis. Variables with statistical significance in univariate analysis were further analyzed in multivariate Cox proportional hazard regression model. Survival curves were plotted according to the Kaplan-Meier method and differences between subgroups were compared using the log-rank test. All tests were two-sided and $P$-values $<0.05$ were considered statistically significant.

\section{Results}

\section{Patient clinicopathologic characteristics}

Baseline clinicopathologic characteristics of all enrolled patients are shown in Table 1. Of these patients, 371 were included in the training cohort and 310 were included in the validation cohort. In the training cohort, the median OS and RFS were 19.4 months and 9.4 months. The OS and RFS rates were $74.1 \%$ and $41.5 \%$ at 1 year, $41.9 \%$ and $22.3 \%$ at 2 years, and $24.2 \%$ and $18.8 \%$ at 3 years, respectively. There were 224 males and 147 females, with a median age of 62 years (range, 35-84). A total of 223 patients had pancreatic head cancer, whereas the remaining had tumors located at the body or tail of the pancreas. According to the AJCC 8th edition, 150 patients were diagnosed with stage I tumor, 186 with stage II tumor, and 35 with stage III tumor. A normal level of preoperative serum CA19-9 was observed in 81 patients and 298 patients received postoperative chemotherapy.

In the validation cohort, the median OS and RFS were 25.0 months and 10.9 months. The OS and RFS rates were $85.4 \%$ and $47.5 \%$ at 1 year, $52.5 \%$ and $24.7 \%$ at 2 years, and $26.2 \%$ and $19.2 \%$ at 3 years, respectively. This cohort included 164 males and 146 females, with a median age of 60 years (range, 34-82). There were 156 patients with pancreatic head cancer and 154 patients with pancreatic body or tail cancer. The number of patients classified into stage I, II, and III was 119, 146, and 45, respectively. Elevated preoperative serum CA19-9 was observed in 243 patients and 256 patients received postoperative chemotherapy.

\section{Correlations between SIRI and clinicopathologic parameters}

As shown in Table 1, patients were stratified into low SIRI group $(\leq 0.69)$ and high SIRI group $(>0.69)$ according to the cutoff value of SIRI. High SIRI group was $66.8 \%$ (248 out of 371) in the training cohort and 59.4\% (184 out of 310 ) in the validation cohort. Significant positive correlations were found in the training cohort and further confirmed in the validation cohort between high level of SIRI and male gender $(P=0.011$ and $P=0.002)$, high level of NLR (both $P<0.001$ ), high level of PLR (both $P<0.001$ ), and high level of SII (both $P<0.001$ ). In addition, we also found that patients with high level of SIRI were more likely to have pancreatic head tumor $(P=0.004)$ and advanced TNM stage $(P=0.033)$ in the training cohort.

\section{Prognostic significance of SIRI in the training cohort}

Results of univariate analysis showed that tumor size, lymph node metastasis, TNM stage, tumor differentiation, vascular invasion, preoperative serum CA19-9, postoperative chemotherapy, NLR, PLR, SII, and SIRI were 
Table I Baseline clinicopathologic characteristics of patients in the training cohort and validation cohort

\begin{tabular}{|c|c|c|c|c|c|c|}
\hline \multirow[t]{2}{*}{ Variables } & \multicolumn{3}{|c|}{ Training cohort $(n=37 I)$} & \multicolumn{3}{|c|}{ Validation cohort $(n=3 \mid 0)$} \\
\hline & SIRI $\leq \mathbf{0 . 6 9}$ & SIRI >0.69 & $P$-value & SIRI $\leq \mathbf{0 . 6 9}$ & SIRI >0.69 & $P$-value \\
\hline \multicolumn{7}{|l|}{ Age (years) } \\
\hline$\leq 62$ & 65 & 137 & 0.663 & 75 & 106 & 0.737 \\
\hline$>62$ & 58 & 111 & & 51 & 78 & \\
\hline \multicolumn{7}{|l|}{ Gender } \\
\hline Female & 60 & 87 & 0.011 & 73 & 73 & 0.002 \\
\hline Male & 63 & 161 & & 53 & 111 & \\
\hline \multicolumn{7}{|l|}{ Tumor location } \\
\hline Head & 61 & 162 & 0.004 & 55 & 101 & 0.052 \\
\hline Body or tail & 62 & 86 & & 71 & 83 & \\
\hline \multicolumn{7}{|l|}{ Tumor size $(\mathrm{cm})$} \\
\hline$\leq 4$ & 91 & 176 & 0.543 & 94 & 129 & 0.387 \\
\hline$>4$ & 32 & 72 & & 32 & 55 & \\
\hline \multicolumn{7}{|l|}{ Lymph node status } \\
\hline Negative & 73 & 130 & 0.207 & 70 & 85 & 0.105 \\
\hline Positive & 50 & 118 & & 56 & 99 & \\
\hline \multicolumn{7}{|l|}{ TNM stage } \\
\hline 1 & 56 & 94 & 0.033 & 55 & 64 & 0.211 \\
\hline II & 62 & 124 & & 52 & 94 & \\
\hline III & 5 & 30 & & 19 & 26 & \\
\hline \multicolumn{7}{|l|}{ Tumor differentiation } \\
\hline Well to moderate & 87 & 156 & 0.135 & 91 & 115 & 0.075 \\
\hline Poor & 36 & 92 & & 35 & 69 & \\
\hline \multicolumn{7}{|l|}{ Vascular invasion } \\
\hline No & 98 & 199 & 0.898 & 96 & 138 & $0.81 \mathrm{I}$ \\
\hline Yes & 25 & 49 & & 30 & 46 & \\
\hline \multicolumn{7}{|l|}{ Perineural invasion } \\
\hline No & 20 & 31 & 0.322 & 25 & 32 & 0.584 \\
\hline Yes & 103 & 217 & & 101 & 152 & \\
\hline \multicolumn{7}{|l|}{ CAI9-9 (U/mL) } \\
\hline$\leq 37$ & 32 & 49 & 0.170 & 31 & 36 & 0.290 \\
\hline$>37$ & 91 & 199 & & 95 & 148 & \\
\hline \multicolumn{7}{|l|}{ Chemotherapy } \\
\hline No & 19 & 54 & 0.149 & 19 & 35 & 0.369 \\
\hline Yes & 104 & 194 & & 107 & 149 & \\
\hline \multicolumn{7}{|l|}{ NLR } \\
\hline$\leq 2.24$ & 105 & 55 & $<0.001$ & 111 & 50 & $<0.001$ \\
\hline$>2.24$ & 18 & 193 & & 15 & 134 & \\
\hline \multicolumn{7}{|l|}{ PLR } \\
\hline$\leq 100.25$ & 48 & 43 & $<0.001$ & 56 & 35 & $<0.001$ \\
\hline$>100.25$ & 75 & 205 & & 70 & 148 & \\
\hline \multicolumn{7}{|l|}{$\mathrm{PNI}$} \\
\hline$\leq 35.15$ & 70 & $|4|$ & 0.992 & 73 & 116 & 0.365 \\
\hline$>35.15$ & 53 & 107 & & 53 & 68 & \\
\hline
\end{tabular}


Table I (Continued).

\begin{tabular}{|c|c|c|c|c|c|c|}
\hline \multirow[t]{2}{*}{ Variables } & \multicolumn{3}{|c|}{ Training cohort $(n=371)$} & \multicolumn{3}{|c|}{ Validation cohort $(n=3 \mid 0)$} \\
\hline & SIRI $\leq \mathbf{0 . 6 9}$ & SIRI >0.69 & $P$-value & SIRI $\leq \mathbf{0 . 6 9}$ & SIRI >0.69 & P-value \\
\hline SII & & & & & & \\
\hline$\leq 330.30$ & 77 & 31 & $<0.001$ & 77 & 20 & $<0.001$ \\
\hline$>330.30$ & 46 & 217 & & 49 & 164 & \\
\hline
\end{tabular}

Abbreviations: SIRI, systemic inflammation response index; CAI9-9, carbohydrate antigen 19-9; NLR, neutrophil to lymphocyte ratio; PLR, platelet to lymphocyte ratio; PNI, prognostic nutritional index; SII, systemic immune-inflammation index.

prognostic factors for OS. Variables that were associated with RFS included tumor size, lymph node metastasis, TNM stage, preoperative serum CA19-9, postoperative chemotherapy, NLR, PLR, SII, and SIRI (Table 2). A high SIRI score significantly predicted both shorter OS $(\mathrm{HR}=2.001, P<0.001)$ and RFS $(\mathrm{HR}=1.884, P<0.001)$ (Figure 1). The median OS and RFS were 26.8 and 14.1 months for patients with SIRI $\leq 0.69$ and 16.5 and 7.9 months for patients with SIRI $>0.69$. The 1-, 2-, and 3 -year OS rates for patients with low level of SIRI and patients with high level of SIRI were $89.4 \%, 56.2 \%$, $40.6 \%$ and $65.7 \%, 34.8 \%, 15.4 \%$, respectively, while the 1-, 2-, and 3-year RFS rates were 58.5\%, 35.2\%, 27.8\% and $33.1 \%, 15.9 \%, 13.2 \%$, respectively.

In multivariate analysis for OS, advanced TNM stage (HR $=1.448, \quad P<0.001)$, poor tumor differentiation (HR $=1.319, P=0.042)$, vascular invasion $(\mathrm{HR}=1.363$, $P=0.048$ ), elevated preoperative serum CA19-9 (HR $=1.380$, $P=0.041)$, postoperative chemotherapy $(\mathrm{HR}=0.493$, $P<0.001$ ), and high level of SIRI (HR $=1.655, P=0.010)$ were identified as independent prognostic factors. Multivariate analysis also confirmed that predictors for RFS included advanced TNM stage (HR $=1.359, P=0.001)$, elevated preoperative serum CA19-9 $(\mathrm{HR}=1.433, P=0.014)$, postoperative chemotherapy $(\mathrm{HR}=0.751, P=0.044)$, high level of NLR $(\mathrm{HR}=1.407, P=0.027)$, and high level of SIRI $(\mathrm{HR}=1.463, P=0.030)$ (Table 3$)$.

\section{Prognostic significance of SIRI in the validation cohort}

The prognostic value of SIRI was further verified in an independent validation cohort of 310 patients. The results were similar to those obtained from the training cohort. High level of SIRI was associated with both poorer OS (HR $=2.141$, $P<0.001)$ and RFS $(\mathrm{HR}=1.831, P<0.001)$ (Table 2, Figure 1). The median OS and RFS were 30.6 and 16.7 months for patients with SIRI $\leq 0.69$ and 21.1 and 9.5 months for patients with SIRI $>0.69$, respectively. The 1-, 2-, and 3-year
OS rates for patients with low level of SIRI and patients with high level of SIRI were $89.6 \%, 65.6 \%, 40.6 \%$ and $82.0 \%$, $42.0 \%, 10.6 \%$, respectively, while the 1-, 2-, and 3-year RFS rates were $60.7 \%, 34.9 \%, 26.7 \%$ and $38.3 \%, 17.8 \%, 11.5 \%$, respectively. Multivariate analysis revealed that high level of SIRI remained an independent predictor of OS $(\mathrm{HR}=2.114$, $P=0.002)$, together with advanced TNM stage $(\mathrm{HR}=1.472$, $P=0.003)$ and postoperative chemotherapy $(\mathrm{HR}=0.457$, $P<0.001)$. Only high level of SIRI $(\mathrm{HR}=1.766, P=0.003)$ and advanced TNM stage $(\mathrm{HR}=1.437, P=0.001)$ significantly affected RFS after multivariate analysis (Table 3 ).

\section{Prognostic value of SIRI in CAI9-9 negative and early-stage PDAC patients}

We further analyzed the prognostic efficiency of SIRI in CA19-9-negative and early-stage subgroups of patients. CA19-9-negative patients referred to those with normal level of preoperative serum CA19-9 ( $\leq 37 \mathrm{U} / \mathrm{mL})$ and earlystage patients referred to those stratified into stage I according to TNM staging system of the AJCC 8th edition. In the training cohort, we found that a high SIRI score was significantly correlated with poor OS and RFS, both in the CA19-9 negative $(\mathrm{HR}=2.102, P=0.012$ and $\mathrm{HR}=1.936, P=0.017)$ and early-stage subgroups $(\mathrm{HR}=2.247, P<0.001$ and $\mathrm{HR}=1.735$, $P=0.006$ ) (Figure 2). The prognostic significance of SIRI for OS (HR $=2.584, P=0.036$ in CA19-9-negative subgroup and $\mathrm{HR}=1.916, P=0.040$ in early-stage subgroup) and RFS (HR $=2.875, P=0.002$ in CA19-9-negative subgroup and $\mathrm{HR}=1.677, P=0.031$ in early-stage subgroup) remained in the validation cohort (Figure 3 ).

\section{Prognostic value of SIRI in patients who received postoperative chemotherapy}

Kaplan-Meier survival curves for patients who received postoperative chemotherapy stratified by SIRI are shown in Figure 4. In the training cohort, patients with SIRI $>0.69$ had a median OS and RFS of 19.3 months 
Table 2 Univariate analysis for survival of patients in the training cohort and validation cohort

\begin{tabular}{|c|c|c|c|c|}
\hline \multirow[t]{2}{*}{ Variables } & \multicolumn{2}{|l|}{ OS } & \multicolumn{2}{|l|}{ RFS } \\
\hline & HR (95\% Cl) & $P$-value & HR $(95 \% \mathrm{Cl})$ & $P$-value \\
\hline \multicolumn{5}{|l|}{ Training cohort } \\
\hline Age (>62/ $\leq 62$ years) & $1.171(0.914-1.500)$ & 0.211 & $1.058(0.842-1.330)$ & 0.628 \\
\hline Gender (male/female) & $1.283(0.993-1.658)$ & 0.057 & $1.249(0.987-1.580)$ & 0.064 \\
\hline Tumor location (body or tail/head) & $1.065(0.828-1.370)$ & 0.623 & $0.938(0.742-1.186)$ & 0.594 \\
\hline Tumor size $(>4 / \leq 4 \mathrm{~cm})$ & $1.648(1.257-2.159)$ & $<0.001$ & $1.297(1.008-1.670)$ & 0.043 \\
\hline Lymph node status (positive/negative) & $1.366(1.065-1.753)$ & 0.014 & $1.424(1.132-1.790)$ & 0.002 \\
\hline TNM stage (III/II/I) & $1.584(1.291-1.944)$ & $<0.001$ & $1.444(1.201-1.736)$ & $<0.001$ \\
\hline Tumor differentiation (poor/well to moderate) & $1.297(1.004-1.675)$ & 0.047 & $1.187(0.935-1.507)$ & 0.160 \\
\hline Vascular invasion (Yes/no) & $1.481(1.104-1.988)$ & 0.009 & $1.308(0.990-1.729)$ & 0.059 \\
\hline Perineural invasion (Yes/no) & $1.466(1.00 \mathrm{I}-2.148)$ & 0.050 & $1.304(0.919-1.851)$ & 0.138 \\
\hline CAI9-9 (>37/ $\leq 37 \mathrm{U} / \mathrm{mL})$ & $1.420(1.044-1.930)$ & 0.025 & $1.513(1.136-2.104)$ & 0.005 \\
\hline Chemotherapy (Yes/no) & $0.484(0.363-0.644)$ & $<0.001$ & $0.718(0.545-0.946)$ & 0.018 \\
\hline $\operatorname{NLR}(>2.24 / \leq 2.24)$ & $1.678(1.299-2.167)$ & $<0.001$ & I.775 (I.402-2.247) & $<0.001$ \\
\hline PLR $(>\mid 00.25 / \leq 100.25)$ & $1.415(1.054-1.901)$ & 0.021 & $1.468(1.115-1.932)$ & 0.006 \\
\hline $\mathrm{PNI}(>35.15 / \leq 35.15)$ & $1.009(0.788-1.294)$ & 0.941 & $0.832(0.660-1.048)$ & 0.118 \\
\hline SII $(>330.30 / \leq 330.30)$ & $1.555(1.172-2.064)$ & 0.002 & $1.567(1.201-2.034)$ & 0.001 \\
\hline SIRI $(>0.69 / \leq 0.69)$ & $2.001(1.512-2.649)$ & $<0.001$ & $1.884(1.46 \mathrm{I}-2.429)$ & $<0.001$ \\
\hline \multicolumn{5}{|l|}{ Validation cohort } \\
\hline Age $(>62 / \leq 62$ years $)$ & $0.951(0.683-1.324)$ & 0.765 & $0.905(0.69 \mid-1.185)$ & 0.467 \\
\hline Gender (male/female) & $\mathrm{I} .177(0.85 \mathrm{I}-\mathrm{I} .630)$ & 0.325 & $1.275(0.967-1.664)$ & 0.074 \\
\hline Tumor location (body or tail/head) & $0.761(0.550-1.054)$ & 0.100 & $0.983(0.755-1.279)$ & 0.896 \\
\hline Tumor size $(>4 / \leq 4 \mathrm{~cm})$ & $1.312(0.925-1.862)$ & 0.128 & $1.392(1.045-1.854)$ & 0.024 \\
\hline Lymph node status (positive/negative) & $\mathrm{I} .74 \mathrm{I}(\mathrm{I} .254-2.417)$ & 0.001 & $1.730(1.326-2.258)$ & $<0.001$ \\
\hline TNM stage (III/II/I) & $1.663(1.312-2.108)$ & $<0.001$ & $1.590(1.314-1.925)$ & $<0.001$ \\
\hline Tumor differentiation (poor/well to moderate) & $1.379(0.989-1.923)$ & 0.058 & $1.289(0.980-1.695)$ & 0.069 \\
\hline Vascular invasion (Yes/no) & $1.316(0.917-1.889)$ & 0.136 & $1.559(1.166-2.084)$ & 0.003 \\
\hline Perineural invasion (Yes/no) & $1.772(1.104-2.845)$ & 0.018 & $1.407(0.979-2.020)$ & 0.065 \\
\hline CAI9-9 (>37/ $\leq 37 \mathrm{U} / \mathrm{mL})$ & $1.738(1.112-2.715)$ & 0.015 & $1.603(1.130-2.274)$ & 0.008 \\
\hline Chemotherapy (Yes/no) & $0.406(0.279-0.590)$ & $<0.001$ & $0.681(0.484-0.956)$ & 0.026 \\
\hline $\operatorname{NLR}(>2.24 / \leq 2.24)$ & $1.640(1.175-2.290)$ & 0.004 & $1.46 \mid(1.120-1.906)$ & 0.005 \\
\hline PLR $(>100.25 / \leq 100.25)$ & I. $108(0.777-1.58 \mathrm{I})$ & 0.572 & $1.082(0.808-1.450)$ & 0.597 \\
\hline $\mathrm{PNI}(>35.15 / \leq 35.15)$ & $0.902(0.644-1.264)$ & 0.550 & $0.992(0.756-1.304)$ & 0.957 \\
\hline SII $(>330.30 / \leq 330.30)$ & $1.564(1.085-2.255)$ & 0.016 & $1.518(1.132-2.035)$ & 0.005 \\
\hline SIRI $(>0.69 / \leq 0.69)$ & $2.141(1.496-3.064)$ & $<0.001$ & I.83। (I.386-2.4I8) & $<0.001$ \\
\hline
\end{tabular}

Abbreviations: OS, overall survival; RFS, recurrence-free survival; CA19-9, carbohydrate antigen 19-9; NLR, neutrophil to lymphocyte ratio; PLR, platelet to lymphocyte ratio; PNI, prognostic nutritional index; SII, systemic immune-inflammation index; SIRI, systemic inflammation response index.

and 8.4 months, significantly shorter than 28.2 months $(\mathrm{HR}=1.922, P<0.001)$ and 14.5 months $(\mathrm{HR}=1.753$, $P<0.001)$ in the low SIRI group. Similarly, the median OS and RFS of patients with high SIRI in the validation cohort were 23.2 months and 10.0 months, also significantly shorter than 33.5 months $(\mathrm{HR}=2.133, P<0.001)$ and 19.5 months $(\mathrm{HR}=1.640, P=0.001)$ in the low SIRI group.

\section{Discussion}

Despite the rapid progress in surgical technique and adjuvant therapies, most pancreatic cancer patients remain at risk for local recurrence, distant metastasis, and short survival. Cancer-related inflammation is recognized as the seventh hallmark of cancer, and it has a close relationship with carcinogenesis and tumor progression. ${ }^{5}$ Many studies have proved that inflammatory scores based on circulating immune and inflammatory cells, such as NLR and PLR, can improve the accuracy of survival prediction. $^{20,21}$ A novel inflammation-based index, SIRI, first described by Qi et al, showed its prognostic value in advanced pancreatic cancer patients who received palliative chemotherapy. ${ }^{10}$ After that, several studies reported 

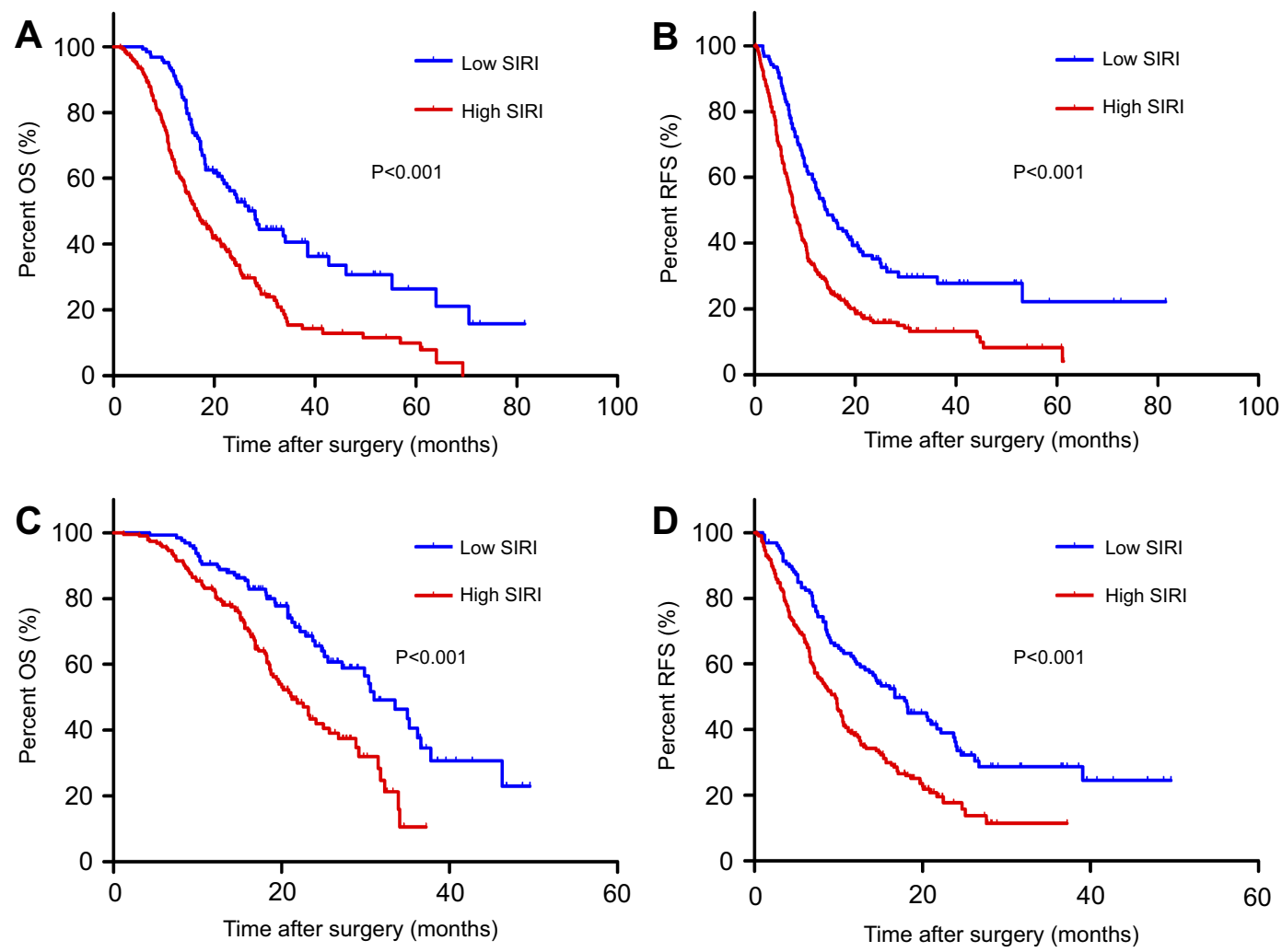

Figure I Prognostic significance of SIRI in PDAC patients undergoing radical surgery. Kaplan-Meier survival curves of OS and RFS stratified by SIRI in the training cohort (A, B) and validation cohort (C, D).

Abbreviations: SIRI, systemic inflammation response index; PDAC, pancreatic ductal adenocarcinoma; OS, overall survival; RFS, recurrence-free survival.

Table 3 Multivariate analysis for survival of patients in the training cohort and validation cohort

\begin{tabular}{|c|c|c|c|c|}
\hline \multirow[t]{2}{*}{ Variables } & \multicolumn{2}{|l|}{ OS } & \multicolumn{2}{|l|}{ RFS } \\
\hline & HR (95\% Cl) & P-value & HR (95\% Cl) & $P$-value \\
\hline \multicolumn{5}{|l|}{ Training cohort } \\
\hline TNM stage (III/II/I) & $1.448(1.180-1.777)$ & $<0.001$ & $1.359(1.132-1.631)$ & 0.001 \\
\hline Tumor differentiation (poor/well to moderate) & $1.319(1.010-1.723)$ & 0.042 & NA & NA \\
\hline Vascular invasion (Yes/no) & $1.363(1.002-1.854)$ & 0.048 & NA & NA \\
\hline CAI9-9 (>37/ $\leq 37 \mathrm{U} / \mathrm{mL})$ & $1.380(1.013-1.880)$ & 0.041 & $1.433(1.075-1.909)$ & 0.014 \\
\hline Chemotherapy (yes/no) & $0.493(0.368-0.660)$ & $<0.001$ & $0.751(0.569-0.992)$ & 0.044 \\
\hline NLR $(>2.24 / \leq 2.24)$ & $1.167(0.835-1.630)$ & 0.366 & $1.407(1.039-1.905)$ & 0.027 \\
\hline PLR $(>100.25 / \leq 100.25)$ & $1.076(0.743-1.557)$ & 0.698 & I.I2I (0.792-I.586) & 0.521 \\
\hline SII $(>330.30 / \leq 330.30)$ & $0.975(0.638-1.490)$ & 0.907 & $0.922(0.624-1.362)$ & 0.683 \\
\hline SIRI $(>0.69 / \leq 0.69)$ & $1.655(1.130-2.425)$ & 0.010 & $1.463(1.037-2.063)$ & 0.030 \\
\hline \multicolumn{5}{|l|}{ Validation cohort } \\
\hline TNM stage (III/II/I) & 1.472 (1.139-1.902) & 0.003 & 1.437 (I.163-I.776) & 0.001 \\
\hline Vascular invasion (Yes/no) & NA & NA & $1.276(0.935-1.742)$ & 0.124 \\
\hline Perineural invasion (Yes/no) & $1.589(0.985-2.564)$ & 0.058 & NA & NA \\
\hline CAI9-9 (>37/ $\leq 37 \mathrm{U} / \mathrm{mL})$ & $1.385(0.882-2.175)$ & 0.157 & $1.332(0.932-1.904)$ & 0.116 \\
\hline Chemotherapy (Yes/no) & $0.457(0.310-0.674)$ & $<0.001$ & $0.73 \mid(0.5 \mid 5-1.040)$ & 0.081 \\
\hline $\operatorname{NLR}(>2.24 / \leq 2.24)$ & $1.142(0.739-1.765)$ & 0.550 & $0.993(0.691-1.428)$ & 0.971 \\
\hline SII $(>330.30 / \leq 330.30)$ & $0.904(0.566-1.443)$ & 0.672 & $1.063(0.733-1.542)$ & 0.747 \\
\hline SIRI $(>0.69 / \leq 0.69)$ & $2.114(1.326-3.370)$ & 0.002 & $1.766(1.209-2.578)$ & 0.003 \\
\hline
\end{tabular}

Abbreviations: OS overall survival; RFS, recurrence-free survival; CA19-9, carbohydrate antigen 19-9; NLR, neutrophil to lymphocyte ratio; PLR, platelet to lymphocyte ratio; SII, systemic immune-inflammation index; SIRI, systemic inflammation response index; NA, not adopted. 

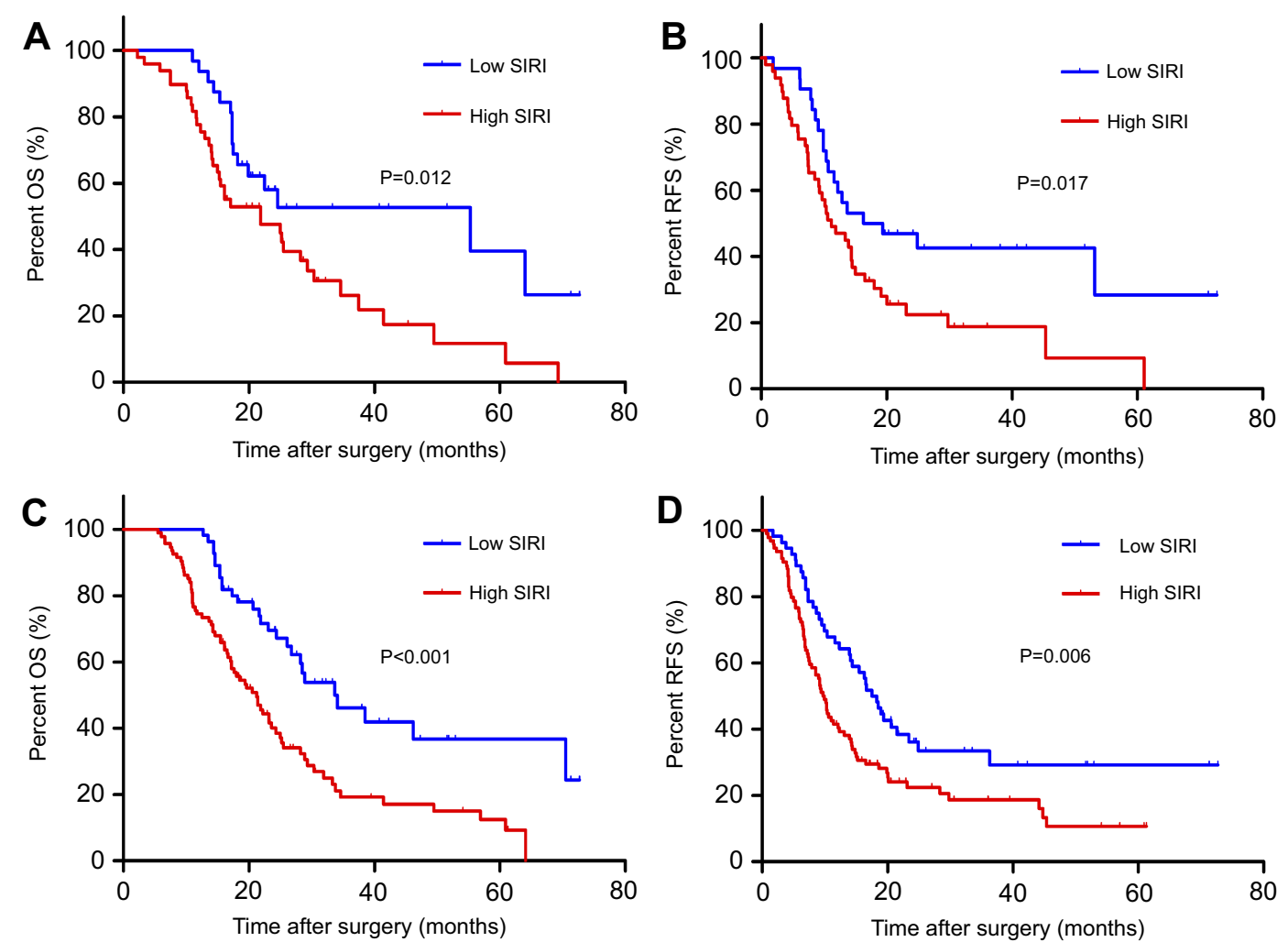

Figure 2 Prognostic significance of SIRI in PDAC patients with normal CAI9-9 and early-stage diseases in the training cohort. Kaplan-Meier survival curves of OS and RFS stratified by SIRI in normal CAI9-9 subgroups (A, B) and early-stage subgroups (C, D).

Abbreviations: SIRI, systemic inflammation response index; PDAC, pancreatic ductal adenocarcinoma; CAI9-9, carbohydrate antigen 19-9; OS, overall survival; RFS, recurrence-free survival.

that SIRI also had prediction ability in esophageal cancer, gastric cancer, and nasopharyngeal cancer. ${ }^{11-13}$ However, there is lack of data to support the significance of SIRI in patients with operable PDAC. In the present study, we demonstrated that SIRI was a significant prognostic indicator for postoperative OS and RFS in PDAC patients after radical surgery in two independent cohorts. Multivariate analysis also identified SIRI as the only significant inflammatory score compared with other scores. Therefore, this preoperative, noninvasive, and easily accessible index has the potential to become a useful biomarker in survival prediction and further helps clinicians make the optimal treatment strategy.

Besides being a prognostic indicator, the index SIRI may also have potential values in guiding individualized therapy in pancreatic cancer. The rapid progress of immunotherapy makes it urgent to find immune-specific biomarkers. Some inflammatory indexes, such as NLR, have been demonstrated to be correlated with the response to immunotherapy in melanoma patients. ${ }^{22}$ Investigation of the potential predictive value of SIRI in pancreatic cancer may help clinicians identify those who are likely to benefit the most from immunotherapy. In addition, neoadjuvant chemotherapy is increasingly being employed for borderline resectable pancreatic cancer. As previously reported, high level of NLR was more frequently observed in pancreatic cancer patients who responded poorly to neoadjuvant chemoradiotherapy compared with those who had positive responses. ${ }^{23}$ This indicates that SIRI may also serve as a candidate biomarker in predicting response to neoadjuvant therapy in patients with pancreatic cancer.

At present, TNM staging is regarded as the gold standard in survival prediction and treatment option. However, precise evaluation of TNM staging can only be obtained after surgery, and prognostic heterogeneity generally exists among patients with the same TNM stage. Increasing evidence suggests that not only the intrinsic histopathologic features of the tumor, but also the host-related factors, are associated with the clinical outcomes of the patients. Combination of TNM staging and SIRI might be important for accurate prediction of patient survival and recurrence. In clinical practice, it is difficult to predict which patient will have recurrence after surgery, especially those with early-stage diseases, such as TNM stage I patients. When 

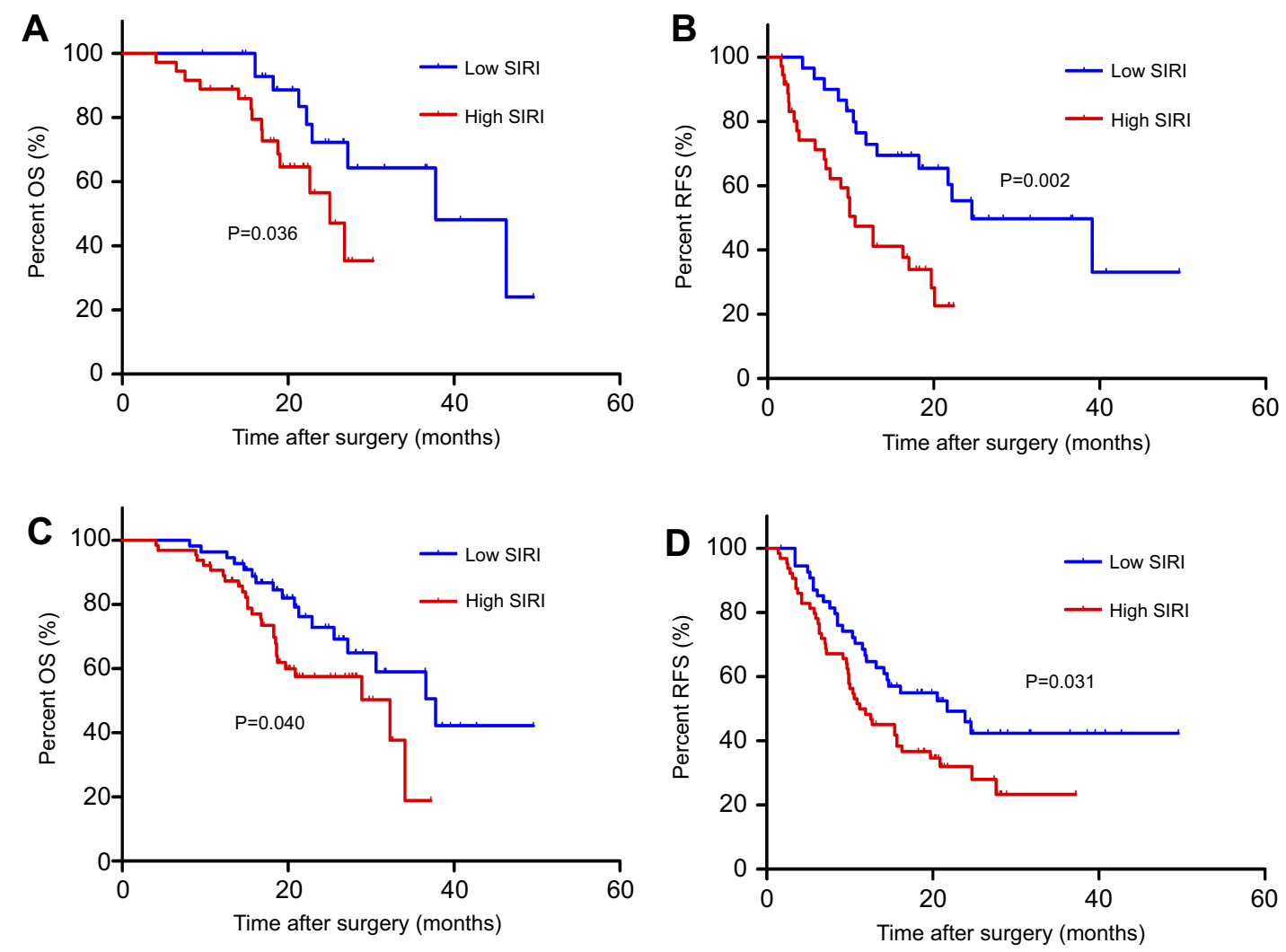

Figure 3 Prognostic significance of SIRI in PDAC patients with normal CAI9-9 and early-stage diseases in the validation cohort. Kaplan-Meier survival curves of OS and RFS stratified by SIRI in normal CAI9-9 subgroups (A, B) and early-stage subgroups (C, D).

Abbreviations: SIRI, systemic inflammation response index; PDAC, pancreatic ductal adenocarcinoma; CAI9-9, carbohydrate antigen 19-9; OS, overall survival; RFS, recurrence-free survival.

stratified patients according to TNM stage, we found that the prognostic ability of SIRI was still strong in stage I patients. CA19-9 is currently the most widely used tumor biomarker in the diagnosis and treatment of pancreatic cancer. However, prognosis for patients with constantly normal level of CA19-9 is usually difficult. We further investigated the prognostic value of SIRI in patients who had normal level of CA19-9 and found that those with higher SIRI had significantly poorer OS and RFS, both in the training cohort and in the validation cohort. Collectively, our results demonstrated that SIRI is a powerful prognostic biomarker, especially for patients with early-stage diseases and nonelevated CA19-9 levels. The clinical utility of SIRI may provide more accurate prognostic information and assist clinicians with postoperative surveillance and management.

For the sake of reducing local recurrence and prolonging survival, postoperative adjuvant chemotherapy has been extensively carried out in the treatment of pancreatic cancer, yet the effect was inconsistently reported among different clinical trials. ${ }^{24-26}$ In our study, we confirmed that it was necessary for patients to receive adjuvant chemotherapy, as adjuvant chemotherapy was an independent protective factor demonstrated by multivariate analysis. On subgroup analysis, the median OS and RFS were both longer in patients with low SIRI compared with patients with high SIRI who had received postoperative chemotherapy. This result was in accordance with a previous report that the improvement in postoperative survival with adjuvant chemotherapy was abolished by persistent inflammatory response. ${ }^{27}$ Identifying appropriate candidates for adjuvant chemotherapy with SIRI may help improve the treatment efficacy and clinical outcome.

Interpretation regarding this study is limited by its retrospective nature and single-center data, which may cause selection bias. The cutoff value of SIRI proposed by our study may not be suitable for other institutions. In addition, we only enrolled patients who had received radical surgery, without considering inoperable patients or the influence of different postoperative chemotherapeutic regimens. Future well-designed, prospective, and 

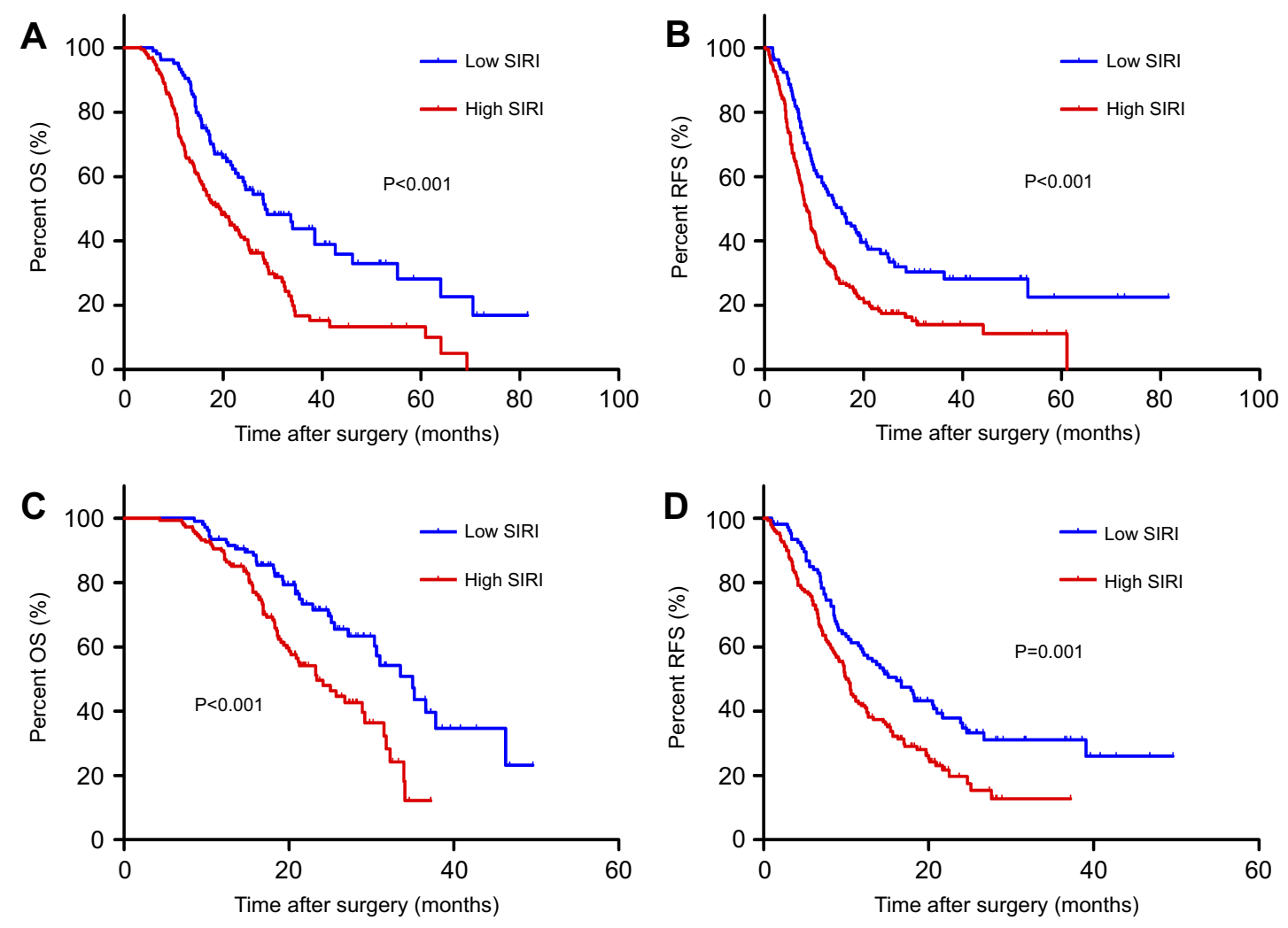

Figure 4 Prognostic significance of SIRI in PDAC patients who received postoperative adjuvant chemotherapy. Kaplan--Meier survival curves of OS and RFS stratified by $\mathrm{SIRI}$ in the training cohort $(\mathbf{A}, \mathbf{B})$ and validation cohort $(\mathbf{C}$,$) .$

Abbreviations: SIRI, systemic inflammation response index; PDAC, pancreatic ductal adenocarcinoma; OS, overall survival; RFS, recurrence-free survival.

randomized controlled clinical trials are requested to further verify the value of SIRI before it can be adopted in routine practice.

\section{Conclusion}

In conclusion, this was the first report to demonstrate that preoperative SIRI qualified as a simple and powerful prognostic indicator in PDAC patients after radical surgery. Clinical utility of SIRI can be helpful in prognostic prediction and treatment response surveillance. Larger prospective studies are needed to verify the preliminary results obtained in the present study.

\section{Abbreviation list}

NLR, neutrophil to lymphocyte ratio; PLR, platelet to lymphocyte ratio; PNI, prognostic nutritional index; SII, systemic immune-inflammation index; SIRI, systemic inflammation response index; PDAC, pancreatic ductal adenocarcinoma; CA19-9, carbohydrate antigen 19-9; AJCC, American Joint Commission on Cancer; OS, overall survival; ROC, receiver operating characteristic; CT, computed tomography; MRI, magnetic resonance imaging; PET-CT, positron emission tomography-computed tomography; RFS, recurrence-free survival; TAMs, tumor-associated macrophages.

\section{Acknowledgments}

This work was supported by grants from the National Science Foundation for Distinguished Young Scholars of China (81625016), the National Natural Science Foundation of China (81472670, 81871941, 81872366, 81802380, and 81802675), the Outstanding Academic Leader Program of the "Technological Innovation Action Plan" in Shanghai Science and Technology Commission (18XD1401200). The funding agencies had no role in study design, data collection and analysis, decision to publish, or preparation of the manuscript.

\section{Disclosure}

The authors report no conflicts of interest in this work.

\section{References}

1. Siegel RL, Miller KD, Jemal A. Cancer statistics, 2018. CA Cancer J Clin. 2018;68(1):7-30. doi:10.3322/caac.21442

2. Chen W, Zheng R, Baade PD, et al. Cancer statistics in China, 2015. CA Cancer J Clin. 2016;66(2):115-132. doi:10.3322/caac.21338 
3. Kamisawa T, Wood LD, Itoi T, Takaori K. Pancreatic cancer. Lancet (London, England). 2016;388(10039):73-85. doi:10.1016/S01406736(16)00141-0

4. Crusz SM, Balkwill FR. Inflammation and cancer: advances and new agents. Nat Rev Clin Oncol. 2015;12(10):584-596. doi:10.1038/ nrclinonc.2015.105

5. Hanahan D, Weinberg RA. Hallmarks of cancer: the next generation. Cell. 2011;144(5):646-674. doi:10.1016/j.cell.2011.02.013

6. Viers BR, Boorjian SA, Frank I, et al. Pretreatment neutrophil-tolymphocyte ratio is associated with advanced pathologic tumor stage and increased cancer-specific mortality among patients with urothelial carcinoma of the bladder undergoing radical cystectomy. Eur Urol. 2014;66(6):1157-1164. doi:10.1016/j.eururo.2014.02.042

7. Grilz E, Posch F, Konigsbrugge O, et al. Association of platelet-tolymphocyte ratio and neutrophil-to-lymphocyte ratio with the risk of thromboembolism and mortality in patients with cancer. Thromb Haemost. 2018;118(11):1875-1884. doi:10.1055/s-0038-1673401

8. Pinato DJ, North BV, Sharma R. A novel, externally validated inflammation-based prognostic algorithm in hepatocellular carcinoma: the prognostic nutritional index (PNI). $\mathrm{Br} J$ Cancer. 2012;106(8):1439-1445. doi:10.1038/bjc.2012.92

9. Hu B, Yang X-R, Xu Y, et al. Systemic immune-inflammation index predicts prognosis of patients after curative resection for hepatocellular carcinoma. Clin Cancer Res. 2014;20(23):6212-6222. doi:10.1158/1078-0432.CCR-14-0442

10. Qi Q, Zhuang L, Shen Y, et al. A novel systemic inflammation response index (SIRI) for predicting the survival of patients with pancreatic cancer after chemotherapy. Cancer. 2016;122 (14):2158-2167. doi:10.1002/cncr.30057

11. Geng Y, Zhu D, Wu C, et al. A novel systemic inflammation response index (SIRI) for predicting postoperative survival of patients with esophageal squamous cell carcinoma. Int Immunopharmacol. 2018;65:503-510. doi:10.1016/j.intimp.2018.10.002

12. Li S, Lan X, Gao H, et al. Systemic Inflammation Response Index (SIRI), cancer stem cells and survival of localised gastric adenocarcinoma after curative resection. J Cancer Res Clin Oncol. 2017;143 (12):2455-2468. doi:10.1007/s00432-017-2506-3

13. Chen Y, Jiang W, Xi D, et al. Development and validation of nomogram based on SIRI for predicting the clinical outcome in patients with nasopharyngeal carcinomas. J Investig Med. 2019;67 (3):691-698. doi:10.1136/jim-2018-000801

14. Mantovani A, Allavena P, Sica A, Balkwill F. Cancer-related inflammation. Nature. 2008;454(7203):436-444. doi:10.1038/nature 07205

15. Kusumanto YH, Dam WA, Hospers GA, Meijer C, Mulder NH. Platelets and granulocytes, in particular the neutrophils, form important compartments for circulating vascular endothelial growth factor. Angiogenesis. 2003;6(4):283-287. doi:10.1023/B:AGEN.00000 29415.62384.ba
16. Muller I, Munder M, Kropf P, Hansch GM. Polymorphonuclear neutrophils and $\mathrm{T}$ lymphocytes: strange bedfellows or brothers in arms? Trends Immunol. 2009;30(11):522-530. doi:10.1016/j. it.2009.07.007

17. Galdiero MR, Garlanda C, Jaillon S, Marone G, Mantovani A. Tumor associated macrophages and neutrophils in tumor progression. $J$ Cell Physiol. 2013;228(7):1404-1412. doi:10.1002/jcp.24260

18. Mantovani A, Schioppa T, Porta C, Allavena P, Sica A. Role of tumor-associated macrophages in tumor progression and invasion. Cancer Metastasis Rev. 2006;25(3):315-322. doi:10.1007/s10555006-9001-7

19. Ferrone C, Dranoff G. Dual roles for immunity in gastrointestinal cancers. J Clin Oncol. 2010;28(26):4045-4051. doi:10.1200/ JCO.2010.27.9992

20. Cheng H, Luo G, Lu Y, et al. The combination of systemic inflammation-based marker NLR and circulating regulatory $\mathrm{T}$ cells predicts the prognosis of resectable pancreatic cancer patients. Pancreatology. 2016;16(6):1080-1084. doi:10.1016/j. pan.2016.09.007

21. Diem S, Schmid S, Krapf M, et al. Neutrophil-to-Lymphocyte ratio (NLR) and Platelet-to-Lymphocyte ratio (PLR) as prognostic markers in patients with non-small cell lung cancer (NSCLC) treated with nivolumab. Lung Cancer. 2017;111:176-181. doi:10.1016/j. lungcan.2017.07.024

22. Ferrucci PF, Gandini S, Battaglia A, et al. Baseline neutrophil-tolymphocyte ratio is associated with outcome of ipilimumab-treated metastatic melanoma patients. $B r \quad J$ Cancer. 2015;112 (12):1904-1910. doi:10.1038/bjc.2015.180

23. Hasegawa S, Eguchi H, Tomokuni A, et al. Pre-treatment neutrophil to lymphocyte ratio as a predictive marker for pathological response to preoperative chemoradiotherapy in pancreatic cancer. Oncol Lett. 2016;11(2):1560-1566. doi:10.3892/ol.2015.4057

24. Kalser MH, Ellenberg SS. Pancreatic cancer. Adjuvant combined radiation and chemotherapy following curative resection. Arch Surg (Chicago, IL: 1960). 1985;120(8):899-903.

25. Klinkenbijl JH, Jeekel J, Sahmoud T, et al. Adjuvant radiotherapy and 5-fluorouracil after curative resection of cancer of the pancreas and periampullary region: phase III trial of the EORTC gastrointestinal tract cancer cooperative group. Ann Surg. 1999;230(6):776-782, discussion 782-774.

26. Neoptolemos JP, Dunn JA, Stocken DD, et al. Adjuvant chemoradiotherapy and chemotherapy in resectable pancreatic cancer: a randomised controlled trial. Lancet (London, England). 2001;358 (9293):1576-1585.

27. Knoop RF, Sparn M, Waldmann J, et al. Chronic pancreatitis and systemic inflammatory response syndrome prevent impact of chemotherapy with gemcitabine in a genetically engineered mouse model of pancreatic cancer. Neoplasia (New York, NY). 2014;16(6):463-470. doi:10.1016/j.neo.2014.05.010
Cancer Management and Research is an international, peer-reviewed open access journal focusing on cancer research and the optimal use of preventative and integrated treatment interventions to achieve improved outcomes, enhanced survival and quality of life for the cancer patient.
The manuscript management system is completely online and includes a very quick and fair peer-review system, which is all easy to use. Visit http://www.dovepress.com/testimonials.php to read real quotes from published authors. 